\title{
Model-independent analysis of inelastic-neutron-scattering data at high momentum transfer
}

\author{
C. Andreani and A. Filabozzi \\ Dipartimento di Fisica e Istituto Nazionale di Fisica della Materia, Università di Roma “Tor Vergata," Via della Ricerca Scientifica 1, \\ Roma, Italy \\ E. Pace \\ Dipartimento di Fisica e Istituto Nazionale di Fisica Nucleare, Università di Roma “Tor Vergata," Via della Ricerca Scientifica 1, \\ Roma, Italy \\ J. Mayers \\ ISIS Facility, Rutherford Appleton Laboratory, Chilton, Didcot, OX11 OQX, United Kingdom
}

(Received 11 March 1996)

\begin{abstract}
A model-independent procedure is proposed which allows us to obtain the asymptotic scaling function at infinite momentum transfer and a comprehensive assessment of deviations of deep inelastic-neutron-scattering data from a perfect scaling behavior, which are due to the finite values of energy and momentum transfers to the target atom. The procedure is applied to simulated and experimental data on polycrystalline $\mathrm{ZrH}_{2}$ at $T=20$ and $290 \mathrm{~K}$. We observe that the impulse approximation, valid for infinite energy and momentum transfers, is not yet completely reached at the finite momentum transfer values $q$ of neutron experiments even at $q \simeq 60 \AA^{-1}$. It is argued that, beyond the specific case of $\mathrm{ZrH}_{2}$, this procedure can be used for the description of deep inelastic-neutron scattering from other molecular and atomic systems. [S0163-1829(96)05333-7]
\end{abstract}

\section{INTRODUCTION}

Until recently inelastic neutron scattering was mainly confined to moderate excitation energies (less than $100 \mathrm{meV}$ $=1200 \mathrm{~K}=800 \mathrm{~cm}^{-1}$ ). The development of spallation neutron sources, such as the IPNS (Intense Pulsed Neutron Source) at Argonne National Laboratory (USA) and the ISIS source at Rutherford Appleton Laboratory (UK) has considerably extended the range of energy transfers which can be accessed by neutron scattering and has opened a new area in condensed-matter research: the study of the dynamics of atomic systems through inelastic-neutron scattering at high momentum transfers $q$ ( $\hbar=1$ units have been used throughout the paper), and energy transfers $\omega .^{1,2}$ Indeed neutron molecular spectroscopy can now be used to study energy transfers ranging over tens of $\mathrm{eV}$ and momentum transfers ranging up to $150 \AA^{-1}$, allowing the momentum distribution of the single atoms, $n(p)$, to be investigated. The technique, known as deep inelastic-neutron scattering (DINS), uses the same principle as the well-known technique of Compton scattering $^{3}$ (which measures electron momentum distributions by the scattering of high-energy photons): at sufficiently high energy and momentum transfers the scattering cross section can be related to the atomic momentum distribution. ${ }^{4}$ Indeed, when the energy and momentum transfers are much higher than the energies and momenta characteristic of the ground state and of the collective behavior of the target, the response function, $S(q, \omega)$, can be related to the momentum distribution of the struck particle by invoking the impulse approximation (IA), which assumes that, at infinite momentum transfers, this particle recoils freely. ${ }^{5}$ High $q$ and $\omega$ can be achieved at the eVS spectrometer operating at the ISIS spallation source, where intense flux of neutrons with electron volt energies are available. ${ }^{6,7}$ However, although high, the $q$ values available are always finite and deviations from the IA can occur: those arising from the interaction of the scattered particle with the surrounding ones, known as final-state effects (FSE) ${ }^{8}$ those coming from the bound nature of the initial state, known as initial-state effects. ${ }^{9}$ Conditions of validity of the IA are still a question of debate and many theories have been developed to calculate in many systems the form and magnitude of deviations from IA arising at finite $q$ values. ${ }^{10-14}$ In some cases deviations from IA are possible even at infinite values of $q$ : for example, for a system of hard spheres it has been shown that the IA regime is never reached, no matter how high the momentum transfer. ${ }^{15}$

For square integrable interaction potentials, it has been rigorously shown ${ }^{16}$ that in the limit of high momentum transfer, the scaling function $F(q, y)$, that is the response function times appropriate kinematic coefficients, depends on a single variable only, the variable $y$ (Ref. 17), and takes the value corresponding to the IA. In the last years the $q$ dependence of the scaling function, its behavior towards the asymptotic regime, $q \rightarrow \infty$, and the extent to which the deviations from IA limit our ability to extract the single-particle momentum distribution in the system under study have been the matter of several experimental (see, e.g., Refs. 18-22) and theoretical (see, e.g., Refs. 10-14,23,24) studies. From the experimental and theoretical work it can be shown that, at the presently accessible momentum transfers, deviations from IA can hinder the extraction of momentum distribution functions. In particular an important question is the size and functional form of these deviations occurring at finite $q$. Various procedures have been proposed in the literature, ${ }^{25-28}$ in order to cope with these deviations, for instance, Sears ${ }^{26}$ suggested 
removing the antisymmetric part of these contributions by simply symmetrizing the data about $y=0$. However, a model-independent framework for a description of DINS in molecular systems is still needed which is also able to cope with the scattering process at finite $q$.

In this paper we propose a model-independent procedure for deriving the asymptotic scaling function at infinite momentum transfer $(q \rightarrow \infty), F(y)$, from DINS experiments in the framework of $y$ scaling. In our method $F(y)$ is derived from a fit performed on a set of experimental data collected in a $q$ range as wide as possible. The fitting procedure also allows us to estimate the form and the magnitude of $y$-scaling violating effects at finite $q$ and it will be shown that the requirement of a wide $q$ range is necessary to isolate $F(y)$ from the scaling violating contributions. Once the asymptotic scaling function has been determined, if IA holds, one can immediately obtain from the asymptotic scaling function the atomic momentum distribution. ${ }^{17}$ In Sec. II the theoretical framework is presented and in Sec. III a brief description of our method is introduced. In Sec. IV the latter is applied, initially to a simulated set of data in a harmonic model for polycrystalline $\mathrm{ZrH}_{2}$, as an internal check for the procedure, and then to real experimental data on the same sample.

\section{THEORETICAL FORMALISM}

The IA is based on two basic assumptions: (i) the inelastic-neutron scattering at high momentum transfers occurs from single atoms, that is the scattering is incoherent, with conservation of energy and momentum, (ii) effects of the final-state interaction between the struck particle and the residual system are neglected. It is then possible to relate the dynamical response function $S(q, \omega)$ to the atomic momentum distribution $n(p)$ via

$$
S(q, \omega)=\int n(\underline{p}) \delta\left(\omega+E_{i}-E_{f}\right) d \underline{p}
$$

where $E_{i}, E_{f}$ are the initial and final energy of the system, respectively. The $\delta$ function in Eq. (1) ensures the energy conservation in the collisions among neutrons and atoms of the target. If $M$ is the mass of the struck atom, $p$ its momentum before collision and $p+q$ its momentum after collision, the final energy can be expressed as

$$
E_{f}=(\underline{p}+\underline{q})^{2} / 2 M+E_{r}(\underline{p}),
$$

where $(p+q)^{2} / 2 M$ is the final kinetic energy of the struck particle $\overline{\operatorname{and}} E_{r}(p)$ is the energy of the residual system. Obviously, the functional form of the momentum distribution in Eq. (1) depends on the interaction acting on the target atoms, and is not necessarily a Gaussian.

In the case of a system of particles which interact through square integrable ${ }^{16}$ or else through confining harmonic potentials, ${ }^{23,29}$ it was shown that the response function of the system, $S(q, \omega)$, approaches exactly the value obtained from the plane-wave impulse approximation when $q \rightarrow \infty$, and that the effects of final-state interactions vanish at least as $1 / q$. By making use of the scaling variable $y$,

$$
y=\left(\omega-q^{2} / 2 M\right) M / q,
$$

it is possible to define a scaling function $F(q, y)$ via its relationship with the response function

$$
F(q, y)=q / M S(q, \omega) .
$$

Then at $q \rightarrow \infty$ the $q$-independent terms in the $\delta$ function in Eq. (1) can be neglected and the asymptotic value of the scaling function becomes

$$
J(y)=\int n(\underline{p}) \delta(y-\underline{p} \hat{q}) d \underline{p},
$$

where $\hat{q}=q / q$.

In the hypothesis of scattering events occurring at high, but finite, momentum transfer, one can assume that the scaling function $F(q, y)$ is regular and therefore can be represented by a series of powers of $1 / q$ :

$$
\begin{aligned}
F(q, y)= & F(y)+F_{(-1)}(y) / q+F_{(-2)}(y) / q^{2}+F_{(-3)}(y) / q^{3} \\
& +O\left(1 / q^{4}\right) .
\end{aligned}
$$

It can be noted that Eq. (6) is valid provided the $F(q, y)$ has a limit and is analytic in $q$ when $q \rightarrow \infty$. The first $q$-independent term in the right-hand side of Eq. (6) represents the asymptotic value for $q \rightarrow \infty$ of the scaling function, whereas the other $q$-dependent terms include the $y$-scaling violating effects, such as FSE. If IA holds, the $q$-independent term in Eq. (6) can be identified with its IA counterpart, i.e., $F(y)=J(y)$ with $J(y)$ defined by Eq. (5). At high momentum transfer the terms $F_{(-n)}(y) / q^{n}$, with $n \geqslant 3$, go rapidly to zero and it is therefore expected that scaling violating effects are entirely described by the linear and the quadratic terms in 1/q. Clearly Eq. (6) has a range of applicability wider than IA, i.e., it can be applied even when the IA does not hold and $F(y)$ cannot be identified with $J(y)$. An expansion like that in Eq. (6) was introduced in Ref. 25, without proof of its convergence. The experimental validity of the convergence of this expansion was investigated in Ref. 30 in the case of electron scattering off finite nuclei and nuclear matter, and in Ref. 27 for the neutron scattering from polycrystalline $\mathrm{D}_{2} \mathrm{O}$ at $T=20 \mathrm{~K}$. In both cases the experimental scaling function was plotted versus $1 / q$ for fixed values of $y$ and a linear behavior of the data for small values of $1 / q$ was observed. In this paper we will extend this kind of analysis, including both linear and quadratic terms of the expansion in Eq. (6).

It has to be pointed out that, in the case of asymptotic series, an expansion like Eq. (6) can be useful even if it does not converge. For example, this is the case for a system of particles interacting through confining harmonic forces, as it was shown in Ref. 29.

\section{DATA ANALYSIS PROCEDURE}

In a DINS experiment epithermal neutron beams are used in order to approach the high momentum transfer region required by the IA. However, if one aims to derive not only the atomic kinetic energy of the investigated system but also the detailed shape of atomic momentum distribution function, it is necessary to separate the asymptotic scaling function from the contributions of the linear and quadratic terms in the $1 / q$ expansion. To this end one has to collect data in a $q$ 
range as wide as possible. Indeed, this allows us to study, together with the scattering at very high $q$, the approach from lower $q$ values to this region, which is determined by the $q$-dependent terms in Eq. (6). Let us assume that the experimental data are represented by the following expression:

$$
F_{R}(q, y)=\int_{-\infty}^{\infty} F\left(q, y^{\prime}\right) R\left(q, y-y^{\prime}\right) d y^{\prime},
$$

where $F(q, y)$ is the experimental scaling function that we assume being described according to Eq. (6) and $R(q, y)$ is the experimental resolution function.

In order to study the contribution of the $F_{(-n)}(y)$ terms to the scattering data, we propose the following procedure: (1) measure the scaling function in the widest possible interval of momentum transfer; (2) express $F(y), F_{(-1)}(y)$, and $F_{(-2)}(y)$ as an overlap of a complete set of functions conveniently truncated; for example we can express $F(y)$, $F_{(-1)}(y)$, and $F_{(-2)}(y)$ as a Gaussian times polynomials, depending on some parameters to be determined (the Gaussian variance and the polynomial coefficients); (3) assume $R(q, y)$ to be a known function, for example a Voigt function (see the following section); (4) use the function $F_{R}(q, y)$, expressed according to Eq. (7), with $F(q, y)$ given by Eq. (6), for a fit of the experimental data.

The parameters derived from the fit will allow us to reconstruct the individual contributions, $F(y), F_{(-1)}(y)$, and $F_{(-2)}(y)$, i.e., the asymptotic scaling function and the $y$-scaling violating effect contributions. The experimental data at highest $q$ values will allow us to better determine $F(y)$, while the data at the lowest $q$ values, the form for $F_{(-1)}(y)$ and $F_{(-2)}(y)$. The fit has to be performed with the normalization condition

$$
\int_{-\infty}^{\infty} F(q, y) d y=1,
$$

which holds for any value of $q$ at high momentum transfer. As a consequence, the following conditions obviously have to be satisfied:

$$
\begin{gathered}
\int_{-\infty}^{\infty} F(y) d y=1, \\
\int_{-\infty}^{\infty} F_{(-1)}(y) d y=0, \\
\int_{-\infty}^{\infty} F_{(-2)}(y) d y=0 .
\end{gathered}
$$

Let us express, $F(y), F_{(-1)}(y)$, and $F_{(-2)}(y)$, as suggested before, as a product of a Gaussian times polynomial as follows:

$$
\begin{gathered}
F(y)=\exp \left(-b y^{2}\right)\left[a_{0}+a_{1} y^{2}\right], \\
F_{(-1)}(y)=\exp \left(-b y^{2}\right)\left[a_{2} y+a_{3} y^{3}\right], \\
F_{(-2)}(y)=\exp \left(-b y^{2}\right)\left[a_{4}+a_{5} y^{2}+a_{6} y^{4}+a_{7} y^{6}\right] .
\end{gathered}
$$

In these equations we have considered $F(y)$ and $F_{(-2)}(y)$ to be even functions of $y$ and $F_{(-1)}(y)$ to be an odd function of $y$ according to Ref. 26. The condition expressed by Eq. (10) is automatically satisfied, while from Eqs. (9) and (11) it follows that the parameters $a_{0}$ and $a_{4}$ can be obtained from the other parameters by the following equations:

$$
\begin{gathered}
a_{0}=\sqrt{\frac{b}{\pi}}-\frac{a_{1}}{2 b}, \\
a_{4}=-a_{5} \frac{1}{2 b}-a_{6} \frac{3}{4 b^{2}}-a_{7} \frac{15}{8 b^{3}} .
\end{gathered}
$$

Equations (15) and (16) imply that in our procedure only the parameters $b, a_{1}, a_{2}, a_{3}, a_{5}, a_{6}, a_{7}$ are to be determined by fitting the whole set of experimental data using the $F_{R}(q, y)$ functional form given in Eq. (7), where $F(q, y)$ is described by Eq. (6) and $R(q, y)$ is the appropriate resolution function.

\section{DATA ANALYSIS OF $\mathrm{ZrH}_{2}$}

We will apply the above procedure to both simulated and experimental data for a sample of polycrystalline $\mathrm{ZrH}_{2}$ and the following notations will be used: functions $F(y)$, $F(q, y)$, and $F_{R}(q, y)$ will have a superscript $s$ or ex when referring to simulated or experimental data, respectively; the functions resulting from the fit will be referred to by a further superscript Fit. Polycrystalline $\mathrm{ZrH}_{2}$ has been chosen because there is experimental evidence that it is an harmonic system. ${ }^{31}$

\section{A. Analysis of the simulated data}

Simulated data for the $F^{s}(q, y)$ scaling function in $\mathrm{ZrH}_{2}$ can be derived by calculating the dynamical structure factor $S(q, \omega)$ for an isotropic harmonic solid. The simulation, based on measurement of the vibrational density of states and within the harmonic incoherent approximation is fully described in Ref. 32. Scaling functions, $F^{s}(q, y)$, were derived at the same scattering angles for which the experiment for $\mathrm{ZrH}_{2}$ on eVS was also performed. ${ }^{32}$ Subsequently, to obtain $F_{R}^{s}(q, y), F^{s}(q, y)$ functions have been convoluted, for each detector, with a resolution function, $R(q, y)$, determined according to a method described in Ref. 18 (see Table I). Following the procedure described in Sec. III a simultaneous fit of the data, for ten angles listed in Table I, was performed in the range $-20 \AA^{-1}<y<20 \AA^{-1}$, for a total number of 1150 points, using for the scaling function the expression given in Eq. (6). Numerical values for the best parameters resulting from the fit and the corresponding values for the $\chi^{2}$ are listed in Table II. It has to be noted that these parameters have been obtained by imposing $a_{1}=0$ in Eq. (12), after having checked that the $\chi^{2}$ value does not significantly change when $a_{1}$ is let free. Furthermore, from Table II we observe that $a_{6}$ and $a_{7}$ are small, so that the terms $a_{6} y^{4}$ and $a_{7} y^{6}$ contribute significantly to Eq. (14) only for $|y|>5 \AA^{-1}$. An important result of our analysis is that the best-fit value obtained for the parameter $b$, $b=0.0288 \pm 0.0002 \AA^{2}$, (see Table II) is practically coincident with the value corresponding to the model used for 
TABLE I. Parameters describing the resolution function, for mass $M=1 \mathrm{amu}$, at the scattering angles of the simulated and experimental data; the variance, $\sigma_{G}$, of the Gaussian component and the half width at half maximum, $\Gamma / 2$, of the Lorentzian component of the Voigt resolution function, $R(q, y)$, are reported for each angle, together with the momentum transfer corresponding to the maximum of each recoil peak (Ref. 18).

\begin{tabular}{cccc}
\hline \hline $\begin{array}{c}2 \theta \\
(\text { degree })\end{array}$ & $\begin{array}{c}\sigma_{G} \\
\left(\AA^{-1}\right)\end{array}$ & $\begin{array}{c}\Gamma / 2 \\
\left(\AA^{-1}\right)\end{array}$ & $\begin{array}{c}\mathrm{q} \\
\left(\AA^{-1}\right)\end{array}$ \\
\hline 36.0 & 0.61 & 0.98 & 35.4 \\
38.1 & 0.60 & 0.91 & 38.3 \\
39.9 & 0.59 & 0.86 & 40.8 \\
41.9 & 0.58 & 0.80 & 43.8 \\
43.7 & 0.58 & 0.75 & 46.8 \\
45.9 & 0.57 & 0.70 & 50.4 \\
47.8 & 0.57 & 0.65 & 53.8 \\
49.7 & 0.56 & 0.61 & 57.6 \\
51.7 & 0.56 & 0.57 & 61.7 \\
53.7 & 0.55 & 0.53 & 66.1 \\
\hline \hline
\end{tabular}
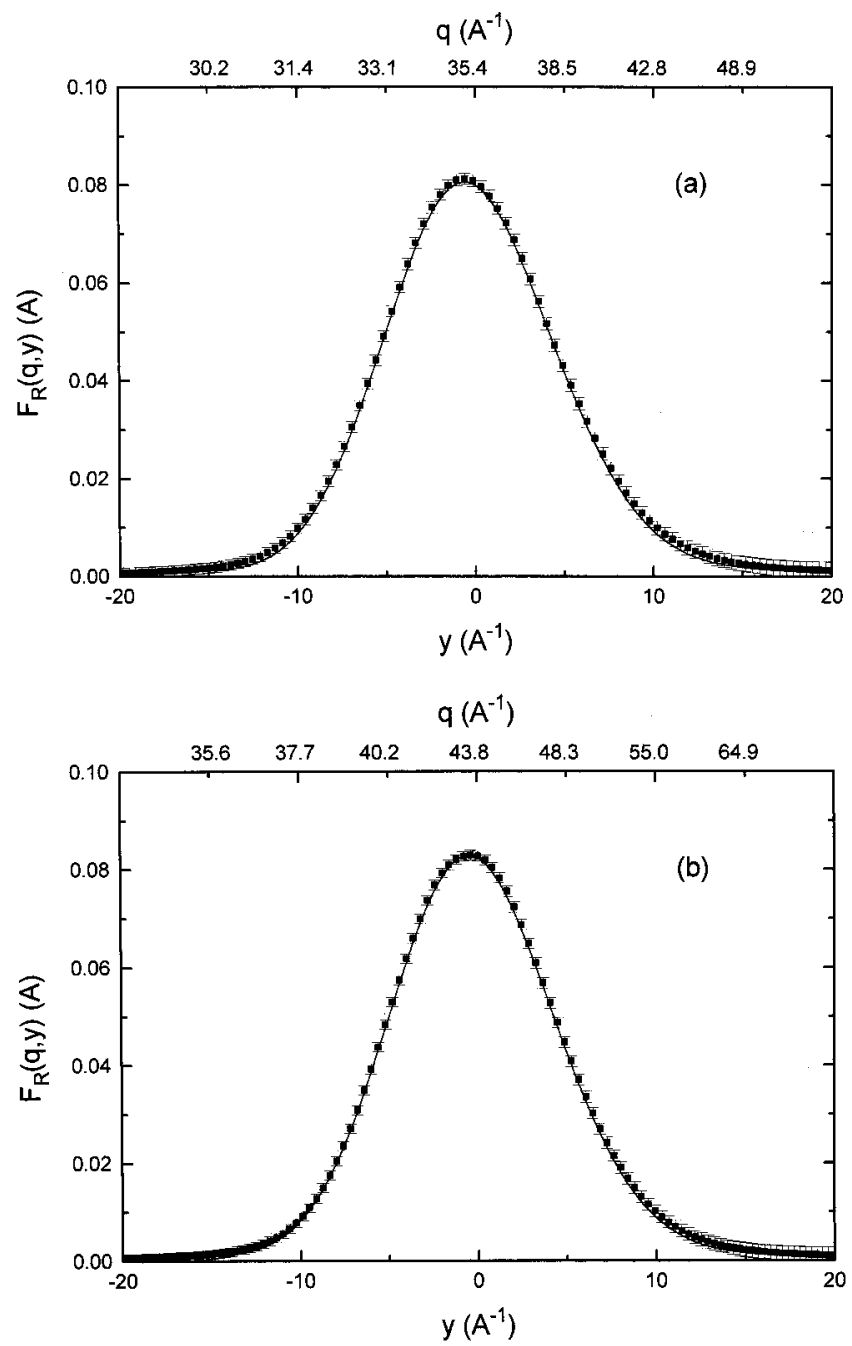

TABLE II. Fitted parameters derived by the simultaneous fit of the $\mathrm{ZrH}_{2}$ simulated and experimental data as described in the text. The relationships between these parameters and the individual contributions of the scaling function are given in Eqs. (12), (13), and (14). The definition of $\chi^{2}$ is $\chi^{2}=[1 /(N-n)] \Sigma\left[\left(x_{i}-z_{i}\right)^{2} / \varepsilon_{i}^{2}\right]$ where $N$ is the number of experimental or simulated points, $n$ is the number of parameters, $x_{i}$ is $F_{R}^{s}(q, y)$ or $F_{R}^{\mathrm{ex}}(q, y), z_{i}$ is $F_{R}^{\mathrm{Fit}}(q, y)$, and $\varepsilon_{i}$ is the error on the data.

\begin{tabular}{lcc}
\hline \hline & Simulated data & Experimental data \\
\hline$b\left(\AA^{2}\right)$ & $2.88 \times 10^{-2}$ & $3.03 \times 10^{-2}$ \\
$a_{0}(\AA)$ & $9.58 \times 10^{-2}$ & $9.83 \times 10^{-2}$ \\
$a_{1}\left(\AA^{3}\right)$ & 0 & 0 \\
$a_{2}(\AA)$ & $-1.28 \times 10^{-1}$ & $-3.1 \times 10^{-2}$ \\
$a_{3}\left(\AA^{3}\right)$ & $1.66 \times 10^{-3}$ & $-7 \times 10^{-4}$ \\
$a_{4}\left(\AA^{-1}\right)$ & $6 \times 10^{-1}$ & 1.3 \\
$a_{5}\left(\AA^{-1}\right)$ & $1.26 \times 10^{-1}$ & $3 \times 10^{-1}$ \\
$a_{6}\left(\AA^{3}\right)$ & $2 \times 10^{-3}$ & $-6 \times 10^{-3}$ \\
$a_{7}\left(\AA^{5}\right)$ & $-5 \times 10^{-5}$ & $-2 \times 10^{-5}$ \\
$\chi^{2}$ & 0.29 & 1.27 \\
\hline \hline
\end{tabular}
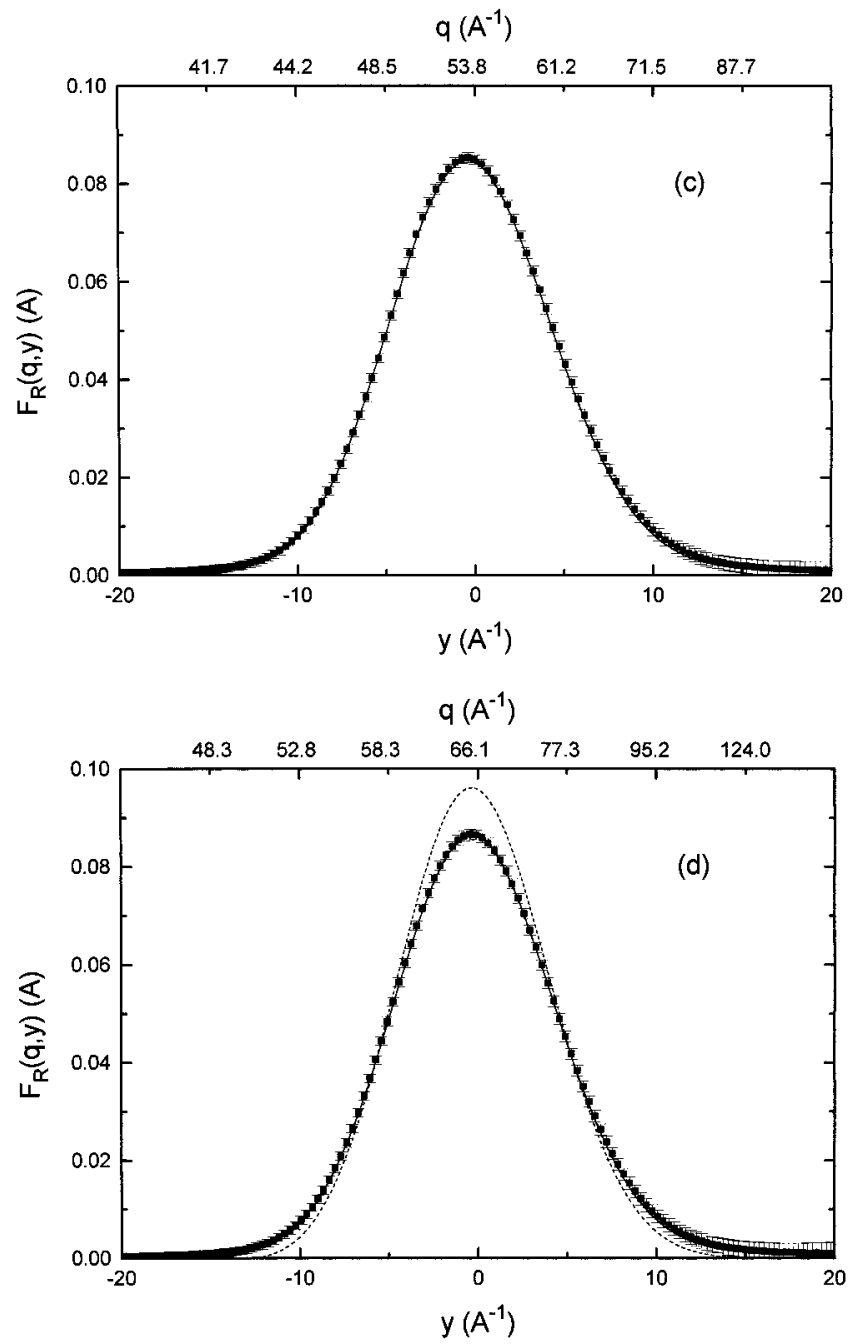

FIG. 1. Simulated $F_{R}^{s}(q, y)$ for $\mathrm{ZrH}_{2}$ at four scattering angles, $2 \theta=36.0^{\circ}, 41.9^{\circ}, 47.8^{\circ}, 53.7^{\circ}$, are plotted as full squares in (a), (b), (c), and (d), respectively. Continuous lines represent the $F_{R}^{s, \text { Fit }}(q, y)$ functions calculated using the fitted parameters listed in Table II. Both simulated and fitted functions are resolution convoluted. The dashed line in (d) represents $F^{s, \text { Fit }}(q, y)$ at the corresponding angle. Lower abscissa is the scaling variable, $y$, and top abscissa the momentum transfer, $q$. 
generating the simulated data $b=1 / 2 \sigma^{2}=0.0287 \AA^{2}$, where $\sigma$ is the Gaussian variance of the asymptotic scaling function.

In Fig. $1 F_{R}^{s, \text { Fit }}(q, y)$ resulting from the fit is compared with $F_{R}^{s}(q, y)$ for four scattering angles $(2 \theta$ $=36.0^{\circ}, 41.9^{\circ}, 47.8^{\circ}, 53.7^{\circ}$. We note that the fit does reproduce the simulated data very well in the whole $q$ range. This implies that the truncation of the series expansion in Eq. (6) up to the $1 / q^{2}$ term is a reasonable assumption. In this figure we also observe that by increasing the scattering angle, i.e., $q$, recoil peak position moves towards $y=0$, while peak intensity increases. These findings originate from two separate contributions, one coming from the explicit $1 / q$ and $1 / q^{2}$ terms in Eq. (6) and the other coming from the $q$ dependence of the resolution function which varies with angle (see Table I). In particular the increase of peak height by increasing $q$ can be mainly ascribed to the better resolution achieved at higher angles. In Fig. 1(d) the $F^{s, \text { Fit }}(q, y)$ is also reported, as a dashed line, to better show the change in intensity specifically due to the resolution contribution at this angle.

In Fig. 2 the $F^{s, \text { Fit }}(q, y)$ functions for three scattering angles $\left(2 \theta=36.0^{\circ}, 41.9^{\circ}, 53.7^{\circ}\right)$ are plotted together with the asymptotic scaling function, $F^{s, \text { Fit }}(y)$, and the individual contributions $(1 / q) F_{(-1)}^{s, \text { Fit }}(y)$ and $\left(1 / q^{2}\right) F_{(-2)}^{s, \text { Fit }}(y)$. We observe that, while recoiling peak moves towards $y=0$ by increasing scattering angles, also some change in peak intensity occurs, both effects being associated with the $1 / q$ and $1 / q^{2}$ contributions. In particular, bearing in mind that $F_{(-1)}(y)$ is an antisymmetric function and $F_{(-2)}(y)$ a symmetric one, we observe that the peak shift comes from the contribution due to the $1 / q$ term in Eq. (6). In addition the $1 / q$ term also produces some distortion in the shape of the recoil peak. From the figure one can see at various angles the individual contributions of $(1 / q) F_{(-1)}^{s, \text { Fit }}(y)$ and $\left(1 / q^{2}\right) F_{(-2)}^{s, \text { Fit }}(y)$, describing the corrections to IA, their magnitude and $q$ dependence. Furthermore in order to show the relevance of the $1 / q^{2}$ term in expansion (6), we repeated the fitting procedure of the simulated data without this term, i.e., by imposing $a_{4}=a_{5}=a_{6}=a_{7}=0$. In this case the quality of the fit is worse ( $\chi^{2}$ becomes 0.45 ) and the parameter $b$ becomes $0.0291 \pm 0.0001 \AA^{2}$, no more so close to the expected value $b=0.0287 \AA^{2}$.

The excellent agreement between simulated data and the fit shown in Fig. 1 at each $q$ value and in the asymptotic limit implies the reliability of the procedure we have used to derive the $F(y)$ function and allows us to apply it to other more general cases. As an example in the next section this procedure will be applied to neutron experimental data of $\mathrm{ZrH}_{2}$.

\section{B. Analysis of the experimental data}

DINS experiment on polycrystalline $\mathrm{ZrH}_{2}$ at $T=20$ and $290 \mathrm{~K}$ were performed using the eVS spectrometer at the ISIS facility at Rutherford Appleton Laboratory (UK). ${ }^{7,32}$ The two sets of data have been analyzed together, since the proton is effectively restricted to the ground state at both these temperatures. ${ }^{32}$ The eVS is an inverse geometry instrument operating for inelastic-neutron-scattering experiments with incident energies in the range $1-100 \mathrm{eV}$. The scattered neutrons are analyzed using a resonance absorption foil (e.g., gold) which defines the final neutron energies. Details about
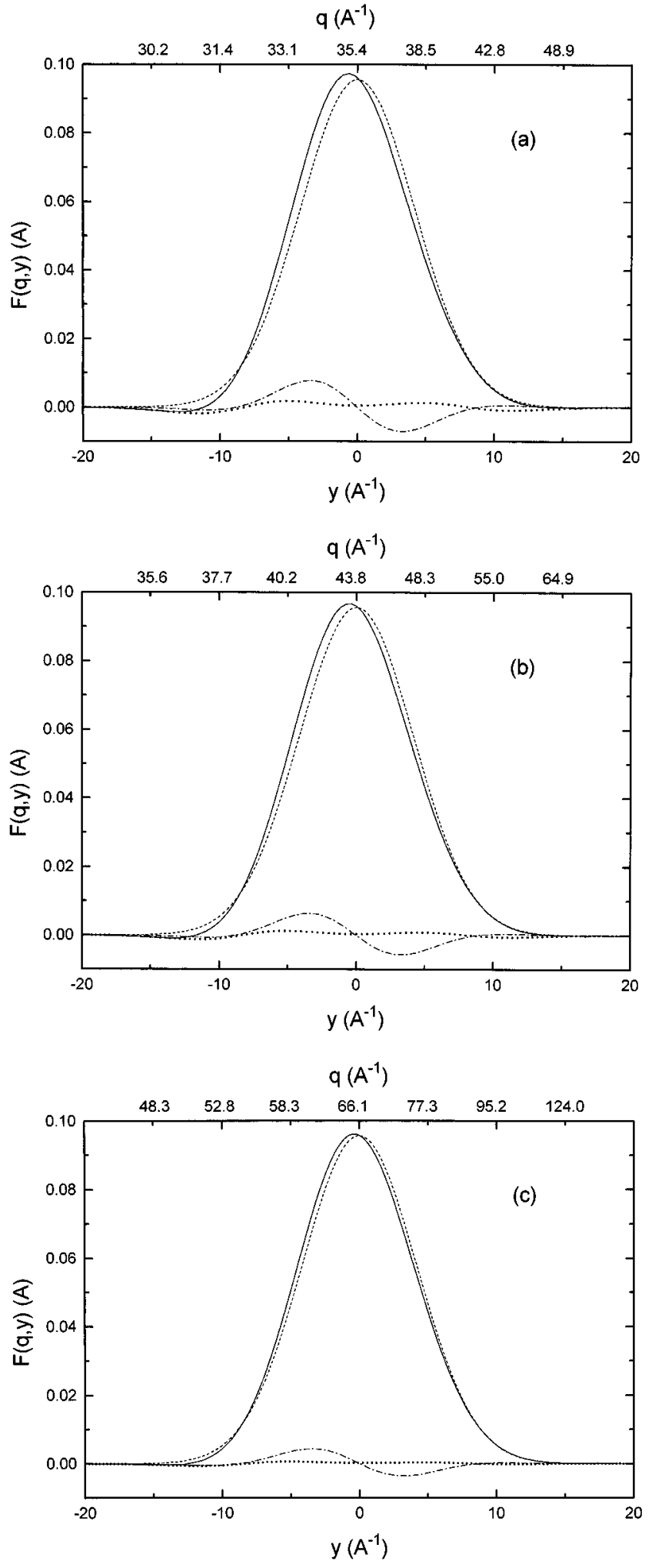

FIG. 2. $F^{s, \text { Fit }}(q, y)$ (continuous line) is compared with $F^{s, \text { Fit }}(y)$ (dashed line), $(1 / q) F_{(-1)}^{s, \text { Fit }}(y) \quad$ (dot-dashed line) and $\left(1 / q^{2}\right) F_{(-2)}^{s, \text { Fit }}(y)$ (dotted line) for $2 \theta=36.0^{\circ}, 41.9^{\circ}, 53.7^{\circ}$, in (a), (b), and (c), respectively. All functions are without the resolution component.

the spectrometer and data analysis procedure are described fully elsewhere. ${ }^{22}$ In this spectrometer different components contribute to the experimental resolution, the most important 

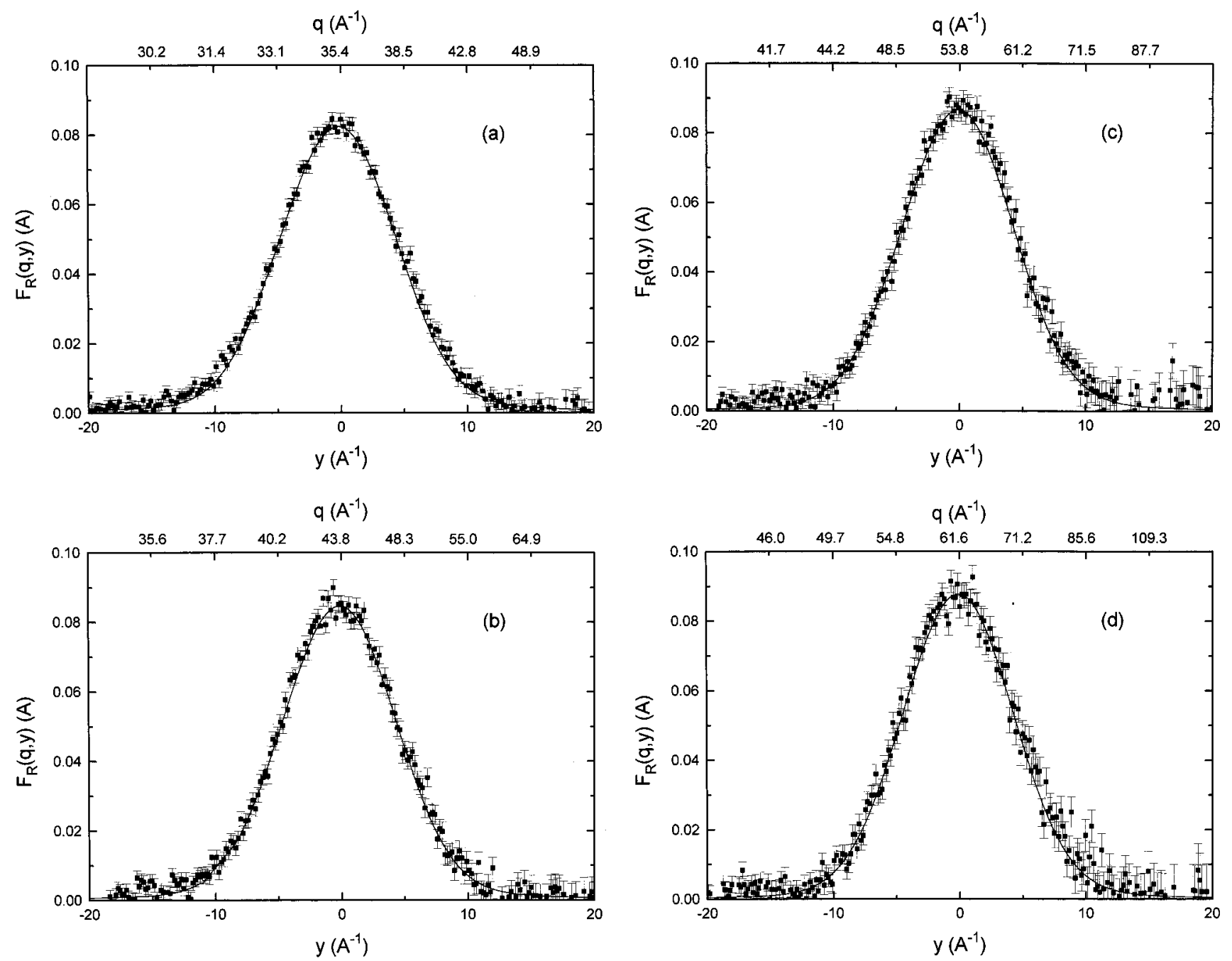

FIG. 3. Experimental $F_{R}^{\mathrm{ex}}(q, y)$ for $\mathrm{ZrH}_{2}$ at four scattering angles. The experimental data for $2 \theta=36.0^{\circ}, 41.9^{\circ}, 47.8^{\circ}, 51.7^{\circ}$ are plotted as full squares in (a), (b), (c), and (d), respectively. Continuous lines represent the $F_{R}^{\mathrm{ex}, \mathrm{Fit}}(q, y)$ functions calculated using the fitted parameters listed in Table II and resolution convoluted.

of which comes from the intrinsic energy width of the resonance foil. The latter is well described by a Lorentzian function and experimentally determined by measuring the recoil scattering from heavy atoms. ${ }^{22}$ The other resolution components, coming from the uncertainties in time and in incident and scattering lengths, are instead well described by Gaussian functions. ${ }^{18}$ Overall the resolution function for the spectrometer is well described by a Voigt function with the individual components at various angles listed in Table I. The same procedure used in the previous section for the analysis of the simulated data is now employed for experimental data at ten scattering angles, in the range $-20<y<20 \AA^{-1}$, for a total number of 2300 points. As previously, a best fit of the data is obtained by allowing just one Gaussian function for $F(y)$ with best fit parameters listed in Table II. We note that the parameter $b$ for the asymptotic scaling function ( $b=0.0303 \pm 0.0004 \AA^{2}$ ) is almost identical to the one corresponding to the simulated data. Therefore one can argue that the harmonic model used for the interaction potential works. As a consequence, the asymptotic scaling function $F(y)$ can be identified with $J(y)$ of Eq. (5) and then the momentum distribution $n(p)$ can be easily derived through

$$
n(p)=-\frac{1}{2 \pi y} \frac{d J(y)}{d y} \quad \text { with } p=|y|
$$

obtaining

$$
n(p)=\frac{a_{0} b}{\pi} \exp \left(-b p^{2}\right) .
$$

In Figs. 3(a), 3(b), 3(c), and 3(d) $F_{R}^{\mathrm{ex}}(q, y)$ for four different scattering angles are compared with the $F_{R}^{\mathrm{ex}, \mathrm{Fit}}(q, y)$ functions. Although the experimental data are somewhat scattered around their fitting values, in particular at high momentum transfer, an overall good agreement is seen on the whole $q$ range. In Fig. 4 the experimental scaling function $F^{\text {ex,Fit }}(q, y)$ is plotted together with the asymptotic scaling function, $F^{\text {ex,Fit }}(y)$, and the individual contributions $(1 / q) F_{(-1)}^{\text {ex,Fit }}(y)$ and $\left(1 / q^{2}\right) F_{(-2)}^{\text {ex,Fit }}(y)$ for three scattering angles $\left(2 \theta=36.0^{\circ}, 41.9^{\circ}, 51.7^{\circ}\right)$. Overall effects similar to those already noted for the simulated data can be observed: a shift of the peak positions towards $y<0$ values for the smaller angles and some contributions from the $q$-dependent terms, even at the highest angles. These effects 

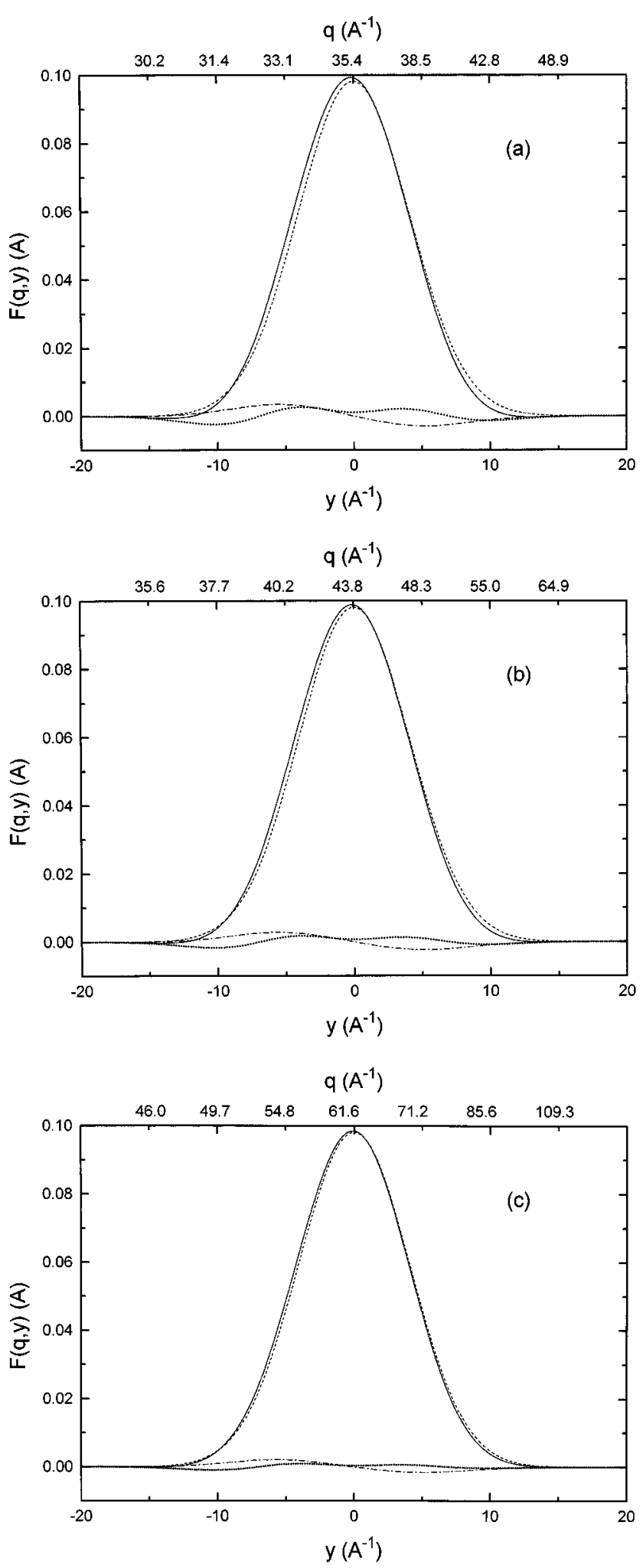

FIG. 4. $F^{\text {ex,Fit }}(q, y)$ (continuous line) is compared with $F^{\text {ex,Fit }}(y)$ (dashed line), $(1 / q) F_{(-1)}^{\text {ex,Fit }}(y)$ (dot-dashed line) and $\left(1 / q^{2}\right) F_{(-2)}^{\text {ex,Fit }}(y)$ (dotted line) for $2 \theta=36.0^{\circ}, 41.9^{\circ}, 51.7^{\circ}$, in (a), (b), and (c), respectively. All functions are without the resolution component.

are a fingerprint of existing deviations from IA, that although small here, are not negligible, even at $q \simeq 60 \AA^{-1}$. Indeed Table II shows that while the parameters pertaining to the $1 / q$ term are smaller, the parameters of the $1 / q^{2}$ term are larger for the experimental data as compared to the simulated ones.

\section{DISCUSSION AND CONCLUSION}

We have shown that a scaling function described by a series expansion in $1 / q$, which incorporate $F(y), F_{(-1)}(y)$, and $F_{(-2)}(y)$ with appropriate Gaussian and polynomial shapes, can be used to fit, in a wide $q$ range, neutronscattering data in the DINS regime. Initially we have applied our procedure to a set of simulated data on $\mathrm{ZrH}_{2}$ and have been able to correctly reproduce the $F(q, y)$ functions at each individual angle; further from the fitted parameters, we have obtained, together with the asymptotic scaling function, both $F_{(-1)}(y)$ and $F_{(-2)}(y)$. The coincidence of the obtained $F(y)$ with the asymptotic scaling function of the harmonic model can be regarded as an internal consistency for our method of analysis, thus indicating that this procedure itself can be applied, in a more general way, to a wide variety of systems with the aim of extracting the asymptotic scaling function from experimental data at finite $q$. When applying our method to a generic system the analysis procedure consists of performing a fit of the experimental data using expansion (6) for the response function; if a good description of the data is obtained, this means that the experimental data do scale and one is entitled to interpret $F(y)$ as the asymptotic scaling function. In the hypothesis that the IA holds [e.g., if the interaction potential satisfies the conditions for identifying $F(y)$ with $J(y)$, see Sec. II] and that the system is isotropic, from the asymptotic scaling function one can then obtain the atomic momentum distribution and the mean kinetic energy of the target atom, $\left\langle E_{k}\right\rangle$, determined as the second moment of $n(p)$. For some systems it can occur that possible forms of the interaction potential are proposed, but a rigorous demonstration of the validity of IA is not available. However, also in these cases one can try to use Eq. (17) and to compare the result with the theoretical momentum distributions obtained from the proposed potentials. In conclusion the domain of applicability of our procedure is a matter that cannot be a priori defined, but has to be checked in any specific case.

Some authors analyze the inelastic-neutron-scattering data by symmetrizing $F_{R}^{\mathrm{ex}}(q, y)$ with respect to $y=0$ in order to retrieve the asymptotic scaling function, in the hypothesis that scaling violating effects are essentially due to the $1 / q$ term and therefore are an odd function of $y .{ }^{26}$ On the contrary in this paper we have shown (see Sec. IV) that the contribution coming from the $1 / q^{2}$ term, although small, in general can not be negligible. Indeed in the case of simulated $\mathrm{ZrH}_{2}$ data the absence of the $1 / q^{2}$ term in Eq. (6) leads to an asymptotic scaling function with a variance different from that of the harmonic model.

Once the $F_{(-1)}(y)$ and $F_{(-2)}(y)$ functions have been obtained applying the procedure described in this paper, a separate question arises about the physical interpretation of both $1 / q$ and $1 / q^{2}$ terms in the series expansion. However, this is beyond the aim of the present work and is a matter which needs to be addressed depending on the specific system under study. 
${ }^{1}$ C.G. Windsor, Pulsed Neutron Scattering (Taylor and Francis, London, 1985).

${ }^{2}$ G.H. Lander and D.L. Price, Phys. Today 38 (1), 38 (1985).

${ }^{3}$ B. Williams, Compton Scattering (McGraw-Hill, New York, 1977).

${ }^{4}$ P.C. Hohenberg and P.M. Platzmann, Phys. Rev. 152, 198 (1966).

${ }^{5}$ S.W. Lovesey, Theory of Neutron Scattering from Condensed Matter (Oxford University Press, London, 1987), Vol. 1.

${ }^{6}$ J. Mayers and A.C. Evans (unpublished).

${ }^{7}$ A.C. Evans, J. Mayers, D.N. Timms, and M.J. Cooper, Z. Naturforsch. 48 A, 425 (1993).

${ }^{8}$ V.F. Sears, Phys. Rev. A 5, 452 (1971); 7, 340 (1973).

${ }^{9}$ J. Mayers, C. Andreani, and G. Baciocco, Phys. Rev. B 39, 2022 (1989).

${ }^{10}$ S. Stringari, Phys. Rev. B 35, 2038 (1987).

${ }^{11}$ A.S. Rinat, Phys. Rev. B 36, 5171 (1987).

${ }^{12}$ R.N. Silver, Momentum distributions, edited by R.N. Silver and P.E. Sokol (Plenum, New York, 1989), p. 111.

${ }^{13}$ C. Carraro and S.E. Koonin, Phys. Rev. Lett. 65, 2792 (1990).

${ }^{14}$ A. Belic and V.R. Pandharipande, Phys. Rev. B 45, 839 (1992).

${ }^{15}$ J.J. Weinstein and J.W. Negele, Phys. Rev. Lett. 49, 1016 (1982).

${ }^{16}$ E. Pace, G. Salme', and G.B. West, Phys. Lett. B 273, 205 (1991).

${ }^{17}$ G. B. West, Phys. Rep. 18, 263 (1975).

${ }^{18}$ C. Andreani, G. Baciocco, R.S. Holt, and J. Mayers, Nucl. In- strum. Methods Phys. Res. Sect. A 276, 297 (1989).

${ }^{19}$ H.A. Mook, Phys. Rev. Lett. 55, 2452 (1985).

${ }^{20}$ D.A. Peek, I. Fujita, M.C. Schmidt, and R.O. Simmons, Phys. Rev. B 45, 9680 (1992).

${ }^{21}$ C. Andreani, A. Filabozzi, M. Nardone, F. P. Ricci, and J. Mayers, Phys. Rev. B 50, 12744 (1994).

${ }^{22}$ J. Mayers, T.M. Burke, and R.J. Newport, J. Phys. Condens. Matter 6, 641 (1994).

${ }^{23}$ J.M.F. Gunn, C. Andreani, and J. Mayers, J. Phys. C 19, L835 (1986).

${ }^{24}$ C. Andreani, A. Filabozzi, and E. Pace, Phys. Rev. B 51, 8854 (1995).

${ }^{25}$ H.A. Gersch, L.J. Rodriguez, and P.N. Smith, Phys. Rev. A 5, 1547 (1972).

${ }^{26}$ V.F. Sears, Phys. Rev. B 30, 44 (1984).

${ }^{27}$ C. Andreani, V. Merlo, E. Pace, and P. Postorino, Phys. Lett. A 171, 76 (1992).

${ }^{28}$ H.R. Glyde, Phys. Rev. B 50, 6726 (1994).

${ }^{29}$ E. Pace, G. Salme', and A.S. Rinat, Phys. Lett. B 325, 289 (1994); Nucl. Phys. A 572, 1 (1994).

${ }^{30}$ C. Ciofi degli Atti, E. Pace, and G. Salme', Phys. Rev. C 43, 1155 (1991).

${ }^{31}$ J. Tomkinson, J. Penfold, and S.T. Robertson, (unpublished).

${ }^{32}$ A.C. Evans, D.N. Timms, J. Mayers, and S.N. Bennington, Phys. Rev. B 53, 3023 (1996). 